\title{
Real-time Optical Spectrum Monitoring in Filterless Optical Metro Networks
}

\author{
B. Shariati ${ }^{1}$, M. Ruiz ${ }^{1}$, F. Fresi ${ }^{2}$, A. Sgambelluri ${ }^{3}$, F. Cugini $^{2}$, and L. Velasco ${ }^{1 *}$ \\ ${ }^{1}$ Optical Communications Group (GCO), Universitat Politècnica de Catalunya (UPC), Barcelona, Spain. \\ ${ }^{2}$ CNIT, Pisa, Italy \\ ${ }^{3}$ Scuola Superiore Sant'Anna, Pisa, Italy \\ *Corresponding author: lvelasco@ac.upc.edu
}

\begin{abstract}
Filterless optical networks (FON) have been proposed as a feasible solution for optical metro networks. In addition, as a result of the shorter distance compared to core optical networks, direct-detection optical transmission systems can be used in metro FONs, which reduces costs, as compared to coherent-detection systems. In this work, we present two optical signal tracking approaches for FONs that allow to detect small frequency laser drift problems and enable safely reducing channel spacing. Additionally, we study the most proper resolution for optical spectrum analyzers (OSA) continuously scanning the whole C-band. Proposed approaches are: $i$ ) feature-based tracking and $i i$ ) residual-based tracking. Experiments are carried out to compare the performance of the approaches for both direct-detection and coherent-detection systems, in the case of a single optical signal with no neighboring signals nearby in the spectrum, as well as in the case of a group of optical signals closely allocated in the spectrum. The results show that the residual-based approach enriched with contextual information enables to better track and correct the wavelength drifts before they reach their limits (e.g., $\pm 2.5 \mathrm{GHz}$ ), leading to the guard band reduction for what concerns wavelength drifts.
\end{abstract}

Keywords: Filterless Optical Networks, Spectrum Surveillance, Optical Signal Tracking.

\section{INTRODUCTION}

The development of optical metro networks, where optical connections typically span few hundreds of kms, requires simpler and more cost-effective solutions compared to those used for long-haul networks [1]. In this regard, modulation formats based on the direct-detection technology, e.g., pulse amplitude modulation (PAM), are candidates for metro applications due to their inherent reduced complexity, power and cost, as compared to coherent-detection systems [2].

In parallel, filterless optical networks (FON), which were proposed as a way to interconnect nodes in a drop and waste network architecture [3], have recently attracted significant attention because of their suitability for metro network applications in combination with coherent transceivers [4]. Specifically, a FON architecture, called horseshoe, was evaluated in [5] to interconnect packet routers via $100 \mathrm{~Gb} / \mathrm{s}$ optical connections (lightpaths). In contrast to spectrum switched optical networks (SSON), where the signals are dropped at their destination, in FON they continue to spread over the transmission line, leading to spectrum waste and efficiency penalties. On the other hand, since the operating lightpaths do not pass through filtering nodes, FONs can operate on grids of any size, e.g., $6.25 \mathrm{GHz}$. In fact, FONs could allow the channels to be placed very close to each other aiming to alleviate spectrum waste. The drawback is that (un)intentional frequency laser drift (fLD) of a transponder (TR), possibly due to failure or misconfiguration, might lead to overlap neighboring channels causing undesirable impact such as Bit Error Rate (BER) degradation. This contrasts with SSON, where overlap effects are limited to some specific cases, being in general much more moderate as intermediate nodes are equipped with Wavelength Selective Switches (WSS) that avoid such overlapping, although they might filter part of the optical spectrum of the current signal [6], [7]. In addition, fLD could have a detrimental impact on the optical signal due to detuning with respect to the receiver optical filter. While coherent receivers can easily track the central frequency $(\mathrm{CF})$ of the transmitted signal by evaluating the offset with a local oscillator, directdetection systems detect only the intensity of the optical signal and can hardly fix fLD effects.

Owing to these facts, cost-effective approaches to monitor FONs are needed to allow network operators to take prompt actions in the case of improper operation of a device in their domain. Some current surveillance systems rely on the capabilities of coherent receivers to collect measurements [8]. Regardless of their complexity, these approaches might limit the performance of surveillance systems, which are intended to monitor the whole domain in real-time with the minimum extra cost and complexity. 
With the development of cost-effective optical spectrum analyzers (OSA) with sub-GHz resolution deployable in the optical nodes [9], noticeable opportunities in the development of surveillance platforms based on optical spectra traces might arise. In this regard, optical spectrum monitoring, with the help of cost-effective OSAs was proposed as a novel solution in [10], as well as in [11], [12] together with machine learning (ML) algorithms [13] to monitor the proper operation of lightpaths in SSON; this approach was demonstrated to detect and identify several soft-failures, including fLD, filter-shift and filter tightening. One of the key parameters determining the cost of the OSAs is their resolution, which has a significant impact on the accuracy of optical signal spectrum-based detection and identification solutions; hence, a target OSA resolution should be determined.

The amount of data generated by an OSA is large, e.g., a 100-MHz resolution OSA generates a vector of 40,000 frequency-power pairs while scanning the whole C-band. To reduce the data to be sent to the network controller, data analytics algorithms can be deployed in the network nodes [14]. Such data analytics distribution simplifies network control by limiting the data exchanged to just a notification whenever some event occurs, and it enables the implementation of local and network-wide control loops (see e.g., [15]-[17]).

Note that producing ML algorithms might require from ML models to be trained with large datasets. In the case of training such algorithms with a dataset that does not cover the whole features space, ML models inaccuracies can appear. The authors in [18] proposed a ML-based algorithm life-cycle to facilitate ML deployment, where the training dataset was initially populated based on the results from simulation and lab experiments and, once ML models are generated, ML re-training can be performed after inaccuracies are detected in the field to improve their precision. This was extended in [19], where the concept of Knowledge Management was presented.

Considering the above-mentioned challenges of FONs and the benefits brought by monitoring optical spectrum using OSAs, it is of paramount importance to devise robust and reliable monitoring solutions that allow FONs to exploit both coherent and direct-detection transmission systems while reducing channel spacing. In this paper, we extend our previous work in [20], [21] and present a set of novel signal identification, classification and tracking algorithms for FONs, as well as study the most proper OSA resolutions for their efficient deployment in optical networks. Specifically, the contribution of this paper is three-fold: $i$ ) the signal identification and tracking procedures are generalized that now support both direct and coherent-detection; ii) the extended features-based tracking approach. This approach uses features extracted only from the monitored optical spectrum. Two different methods of the feature-based approach are proposed: the first one uses directly the features extracted from the optical signal, while the other one elaborates on the features and solves some of the issues of the direct approach; and iii) the new residual-based signal tracking approach, which uses a set of residual signals calculated by subtracting the measured signal by the OSA from an expected version of the signal. In this case, in addition to individual signal tracking analysis, the approach is enriched with the capability of using tracking information from neighboring signals to enhance tracking accuracy, in a sort of contextual analysis.

The rest of the paper is organized as follows. The main ideas for optical signal identification, classification, and tracking spectrum based on continuously analyzing the optical spectrum are first introduced in Section 2. Next, two different optical signal tracking approaches are explored in Section 3: feature-based and residualbased tracking. The discussion is supported by the results presented in Section 4, where the performance of our proposals is demonstrated for both PAM-4 and Quadrature Phase Shift Keying (QPSK) transmission systems. Finally, Section 5 draws the main conclusions.

\section{SPECTRUM SURVEILLANCE AND SIGNAL TRACKING IN FONS}

In contrast to SSONs, FONs eliminate or minimize the number of active switching elements in the optical line systems. An example of FON connecting packet nodes is presented in Fig. 1; FON nodes (FN) comprise only passive optical splitters and combiners and they can be exploited to create simple drop and waste network topologies like buses (or horseshoes). However, FONs can be combined with Optical Cross-connects (OXC) (as suggested in Fig. 1) and participate in extending mesh networks based on SSON [22]. 


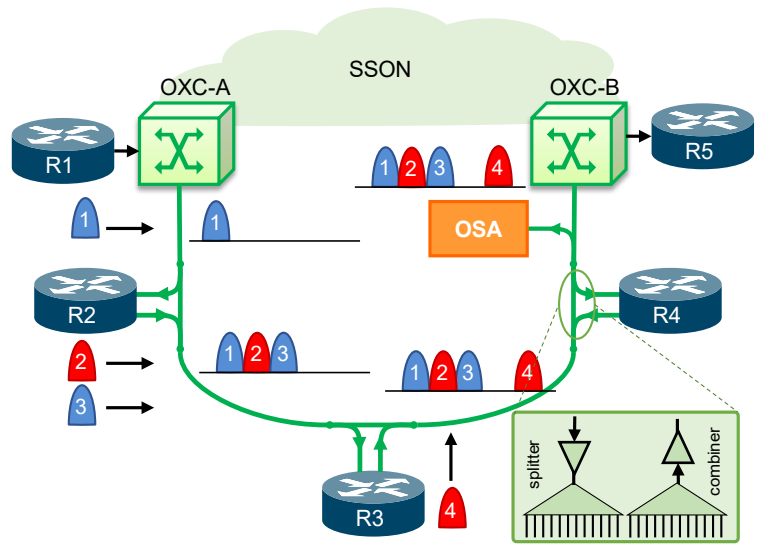

Fig. 1. Example of a horseshoe FON.

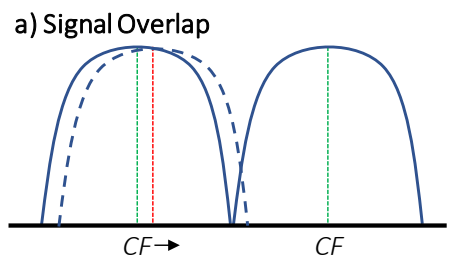

b) Signal misalignment

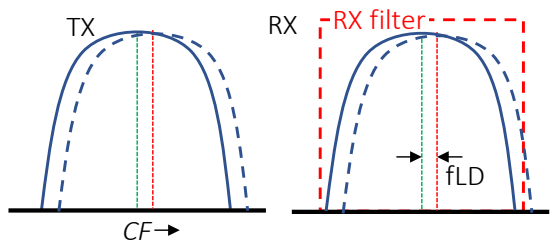

Fig. 2. Signal overlap (a) and misalignment (b)

In FONs, traffic is broadcasted throughout the network; for instance, in Fig. 1 four lightpaths are created: R1$>\mathrm{R} 4$ (labeled 1), R2->R3 (2), R2->R5 (3), and R3->R5 (4). Let us imagine that TRs for lightpaths 2 in R2 and 4 in R3 experience an fLD failure and the signals are shifted to the right in the spectrum. In this case, signal 3 might be affected if the spectrum of signal 2 overlaps it (see Fig. 2a). Therefore, signal overlap is a critical issue for FONs regardless of the transmission technology (i.e., coherent or direct-detection) as it limits channel spacing reduction between neighboring signals. In addition, signals 2 and 4 themselves might be affected if the receivers are not capable of tracking their CFs and misalignment between signals and optical filters at the receivers occur. Therefore, misalignment between the signal and the receiver filter only might happen in directdetection systems. In fact, in PAM-4, a small detuning of the transmitter leads to a noticeable performance penalty at the receiver, as direct-detection receivers are not capable of tracking the laser wavelength of the transmitter; thus, any amount of fLD results in misalignment between the signal launched by the transmitter and the optical filter in the receiver (see Fig. 2b).

An effective solution is to consider a reliable and robust spectrum surveillance and optical signal tracking procedure to detect fLDs accurately, so TRs can be adequately retuned if fLD is detected. Consequently, channel spacing can be reduced while assuring proper TR operation.

The proposed spectrum monitoring approach has an advantage while exploited for FONs, which is the small number of OSAs required for real-time network spectrum monitoring. Specifically, one single OSA installed in the last node of a filterless segment can acquire the optical spectra of all signals in the FON due to the traffic broadcast nature, whereas in a similar SSON, one OSA per link (five in total in Fig. 1) would be required. Acquired spectrum needs to be analyzed real-time so active lightpaths in the FON are monitored and prompt actions are taken before a properly operating lightpath becomes affected by a failed TR. Note that the frequency range of a signal might not be exactly determined and slightly change along lightpaths' lifetime. Therefore, an algorithm examining the captured optical spectrum cannot just select a frequency range in the whole C-band acquired by an OSA and focus on analyzing it in the hope that the whole spectrum of a target lightpath, and only of such lightpath, is confined within that frequency range.

In view of that, we propose to deploy an algorithm in the OXC agents to periodically scan the whole C-band and compare the found signals against the list of lightpaths received from the network controller, which includes relaxed frequency ranges defined by left (LF) and right (RF) frequencies for each one (Fig. 3a). The scan process is intended to ensure that signals in the spectrum and lightpaths in the list match in terms of frequency ranges. Specifically, we aim at checking whether each signal is confined within the expected frequency range allocated to a lightpath (normal signals, like $P_{a}-S_{a}$ in Fig. 3a); conversely, three anomalies can be identified, namely: $i$ ) a signal is partially out of the spectrum allocated to a lightpath (out of range, like $P_{b}-S_{b}$ ); ii) no signal has been detected in the spectrum allocated to a lightpath (missing, like $P_{c}$ ); and iii) a signal is in a spectrum range no allocated to any lightpath (unknown, like $S_{d}$ ). The detection of any of these anomalies triggers a notification with critical severity level to the controller.

Normal signals are then continuously tracked to predict a potential anomaly, i.e., when a signal's CF shifts and might end impacting neighboring signals. In the example in Fig. 3b, although some amount of fLD is detected for signal 2 at time $t_{l}$, the prediction foresees no overlap in the near future with signal 3 . However, at time $t_{2}$, the prediction for signal 2 states that it will exceed its spectrum allocation and thus, a notification with 
warning severity level is triggered towards the controller before an out-of-range anomaly is detected (that actually happens at $t_{3}$ ).

a)

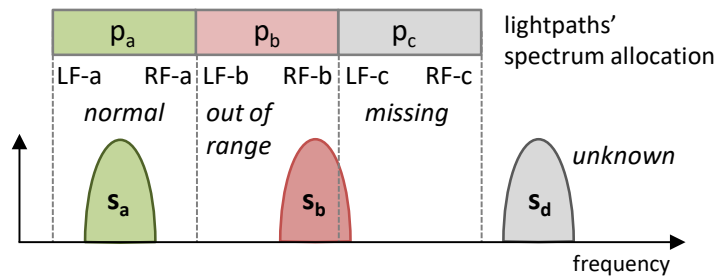

b)
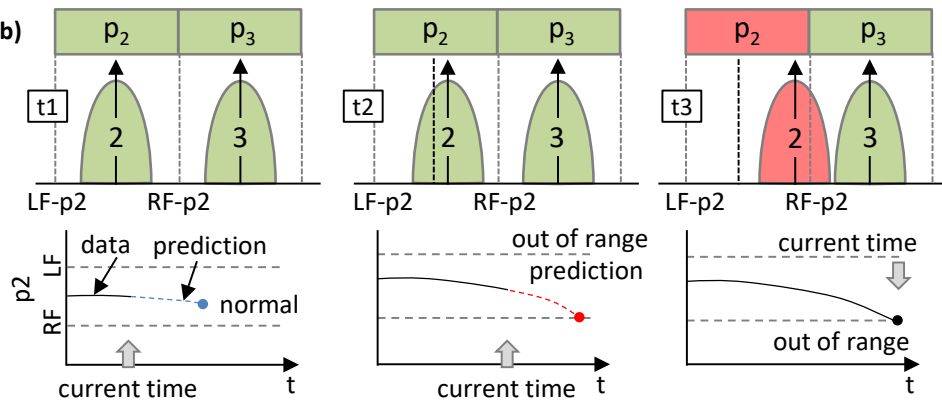

Fig. 3. Signal identification example (a) and signal tracking (b).

\section{OPTICAL SIGNAL TRACKING}

This section focuses on the optical signal tracking function and it proposes two different approaches.

\section{A. Feature-based Signal Tracking}

In this subsection, we propose an optical signal tracking approach that relies on extracting some meaningful features describing key characteristics of the optical spectrum. Here, we consider the Feature Extraction (FeX) module presented in [11] that receives the optical spectrum acquired by an OSA in the form of an ordered list of frequency-power pairs $(\langle f, p>)$ and computes a set of features (or relevant points) to characterize it. The considered features include (see Fig. 4): $i$ ) the range of frequencies where the signal is confined; the left and right frequencies of that range (edges of the signal) are computed using the derivative and are denoted as $\partial$; and ii) a family of power levels computed with respect to the mean $(\mu)$ of the power around the central frequency $(\Delta f)$ minus a number of $\mathrm{dB}$, denoted as $\mathrm{dB}$. Each of these features is represented by a couple of cut-off points denoted as $\left\langle f_{1}(\cdot), p_{I}(\cdot)\right\rangle$ and $\left\langle f_{2}(\cdot), p_{2}(\cdot)\right\rangle$ (note that for a given power level and a normal symmetrical signal, $p_{I}(\cdot) \approx p_{2}(\cdot)$ -slight differences can be caused by OSA's resolution).

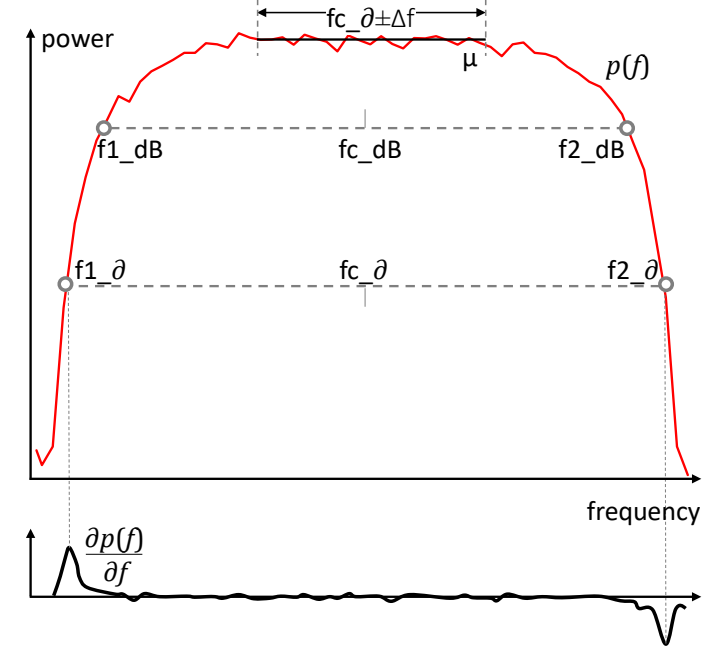

Fig. 4. Some features from a QPSK signal (adapted from [11]).

Considering the above features, we can track an optical signal and measure the amount of fLD that it experiences. Two different methods are devised that exploit those features: $i$ ) Direct Features that relies on the 
features as extracted by the FeX module; and ii) Estimated Features that relies on modeling a fLD estimator using multiple linear regression [24].

In the Direct Features method, the relevant points extracted by the FeX module are used to estimate the fLD and to track the evolution of the signals. fLD is defined as the difference between the expected $C F$ of the signal and the one resulting from the extracted features. More specifically, $f L D_{\text {direct }}=C F_{\text {exp }}-C F_{\text {fea }}$, where $C F_{\text {fea }}=1 / 2$ $\left(f_{1}(\cdot)+f_{2}(\cdot)\right)$, e.g., $C F_{-3 \mathrm{~dB}}=1 / 2\left(f_{1(-3 \mathrm{~dB})}+f_{2(-3 \mathrm{~dB})}\right)$. While this method can be used to estimate the amount of fLD, $p_{I}(\cdot)$ and $p_{2}(\cdot)$ are more relevant to detect signals overlap. In addition, these relevant points can be used to track the evolution of the signal with time and eventually to predict whether it is likely to exceed the spectrum allocation within a given future time window. Although the pre-processing phase of this method is simple, as it just requires the output of the $\mathrm{FeX}$, its accuracy is intrinsically dependent on the OSA resolution.

In the Estimated Features method, the outputs of the FeX module are manipulated to obtain an accurate estimation model for the amount of fLD experienced by a signal. This method requires a dataset with optical spectral samples including various levels of fLDs and the corresponding relative points of each spectrum returned by the FeX module to train a multiple linear regression model that can be later used to estimate the amount of fLD. Specifically, the power levels at $-3 \mathrm{~dB}$ and $-6 \mathrm{~dB}$, as well as the ones computed from the derivative used to compute signal edges can be used. The model can be generalized as follows, where coefficients $b_{i}$ are found during the training phase and $\varepsilon$ denotes an unbiased Gaussian error.

$$
f L D=\sum_{i \in\{-3 d B,-6 d B, \delta\}} b_{i} \times f L D_{i}+b_{0}+\varepsilon \sim \mathcal{N}\left(0, \sigma^{2}\right)
$$

Even though the pre-processing phase of this method becomes a bit more sophisticated compared to the Direct Features one due to the need for the training dataset and large number of required inputs from the FeX module, it performs much better and solves the OSA resolution dependency of the previous method, as it will be shown in Section 4.

\section{B. Residual-based Signal Tracking}

In the residual-based tracking approach, we tackle the problem from another perspective. Our proposal relies on computing an expected version of the signal and compare it against the one acquired by the OSA.

The expected signal is a synthetically generated version of the signal that is expected to be detected by an ideal receiver and it should leave all the noise-related characteristics aside. Although the expected signal could be generated using analytical formulae considering the specifics of every lightpath, i.e., baud rate, shaping-filter in the transmitter, etc. [12], we used the following approach: $i$ ) we start by a square pulse of width equal to the 3$\mathrm{dB}$ bandwidth of the corresponding optical spectrum; ii) we apply a root-raised-cosine (RRC) shaping filter to emulate the shaping filter of the transmitter; iii) in the case the signal traverses some WSSs (as signal 1 in Fig. 1 ), a $2^{\text {nd }}$ order Gaussian filter was applied in the generation of the expected signal before injecting it into the line, which also affects the shape of the spectrum.

The residual is computed by subtracting expected and acquired signals, and it is used as baseline to track the signal under study. Fig. 5 presents the optical spectra for PAM-4 and QPSK, when they are not affected by fLD (Fig. 5a) and with fLD (Fig. 5b). In particular, the carrier in the optical spectrum of PAM-4 signal can be observed [23]. In addition, the residual signal is represented, where its inherent sensitivity to fLD is clearly observed. In consequence, the fLD estimation module requires as inputs the expected shape and the location of every signal in the spectrum.

Let us describe the residual signal procedure, which is similar for QPSK or PAM-4 signals. The measured spectrum and their corresponding expected ones are shown in Fig. 5, where a 1.2-GHz resolution OSA was emulated by averaging power values on windows of such width. The next step is to subtract the expected signal from the acquired one to produce a third signal, named residual. If the measured and expected signal are on the same CF, the residual signal oscillates around 0 (Fig. 5a); conversely, a positive or negative slope, depending on the direction of the drift, is observed (Fig. 5b). For modeling the fLD estimator, portions of left and right edges were considered as features (only the left portion is highlighted in blue Fig. 5 for the sake of clarity); those portions are expected to be the most affected due to the fLD. The final step is to apply multiple linear regression on these features to obtain a model for fLD estimation. 
For PAM-4 signals, we consider portions of $2.4 \mathrm{GHz}$ width, as the maximum expected fLD is small compared to the spectral occupation of the signal, whereas $20.4 \mathrm{GHz}$ portions width can be considered for QPSK signal, as larger fLD can be tolerated. This means that, in the particular case of Fig. 5, where the traces were acquired by a 1.2-GHz resolution OSA, such portions contain just $3<f, p>$ pairs for PAM-4 signals and 18 pairs for QPSK ones. Then, the residual $r$ for each pair can be defined as $\left\langle f, p_{r}=p-p_{e s}\right\rangle$, where $\underline{p}_{e s}$ is the expected signal at frequency $f$. We denote the pairs in the considered left and right portions as $R_{l e f t}$ and $R_{r i g h t}$, respectively, and $R=$ $\underline{R}_{\text {left }} \cup R_{\text {right }}$. Then, the multiple linear regression model for this approach is:

$$
f L D=\sum_{r_{i} \in \mathrm{R}} b_{i} \times r_{i} \cdot p_{r}+b_{0}+\varepsilon \sim \mathcal{N}\left(0, \sigma^{2}\right)
$$

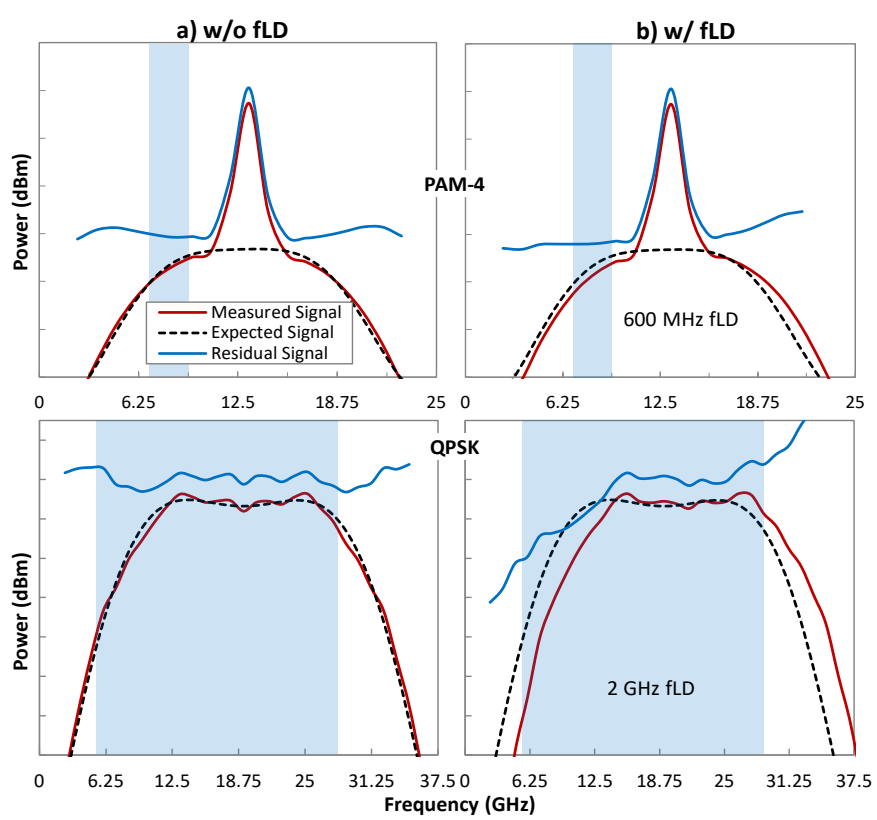

Fig. 5. PAM-4 and QPSK optical spectra captured by a 1.2-GHz resolution OSA, w/o (a) and w/ (b) fLD.

Although the residual-based approach works well when fLD affects one signal but no overlap is caused, it might fail otherwise. An example of this can be seen in Fig. 1, where it would find fLD in signal 3, which is actually a normal signal. To avoid the effects of spectrum overlap, the fLD estimation procedure analyzes the spectrum forward from left to right and considers contextual information, i.e., once it detects fLD in one signal (e.g., in signal 2), it analyzes the following one (e.g., signal 3) considering the actual position of the previous.

The pre-processing phase of this approach is a bit more sophisticated than the previous ones as it requires an additional module to calculate the expected signal for every lightpath, and additionally, it requires a dataset of optical spectra for the multiple linear regression modeling.

\section{PERFORMANCE EVALUATION}

In this section, we first present the setup used to evaluate the performance of the proposed optical signal tracking approaches for both PAM-4 and QPSK signals. Next, we focus on studying the accuracy of the optical signal tracking approaches for individual PAM-4 and QPSK optical signals and finally, the tracking of all the signals in an optical link.

\section{A. Setup description and motivation}

For our experiments, we considered 12.5 GBaud (25 Gb/s) PAM-4 and 30 GBaud polarization multiplexed (PM) QPSK signals. To generate a PAM-4 signal (see the setup in Fig. 6a), the electrical shaping filter of the transmitter is modeled as a $12.5 \mathrm{GHz}$ RRC shaping with mild roll-off factor of 1, driving a Mach-Zehnder Intensity Modulator with $30 \mathrm{~dB}$ extinction ratio. At the receiver, the desired channel is extracted through a 25 $\mathrm{GHz}$ optical filter modeled as in [25]. Optical power at the receiver is $-10 \mathrm{dBm}$ and a post-detection RRC filter with roll- off 1 and $10 \mathrm{GHz}$ bandwidth is applied on the photo-detected signal; thermal noise from photodetectors is also included. BER measurements are performed by means of symbol-by-symbol hard 
threshold detection; no processing is performed on the acquired waveforms prior to BER measures, except timing recovery to find the optimum decision point within the symbol time, as well as the optimum decision thresholds.

QPSK signals were generated with an experimental setup (Fig. 6b), where two neighboring $100 \mathrm{~Gb} / \mathrm{s}$ signals (emulating signals 2 and 3 in Fig. 1) were launched (Fig. 7a). Signal 3 was generated by a commercial system, whereas signal 2 was generated using an experimental system based on Nyquist wavelength division multiplexing, derived from the lab implementation in [26], where a RRC shaping filter with a roll-off of 0.2 and a bandwidth of $15 \mathrm{GHz}$ was used.

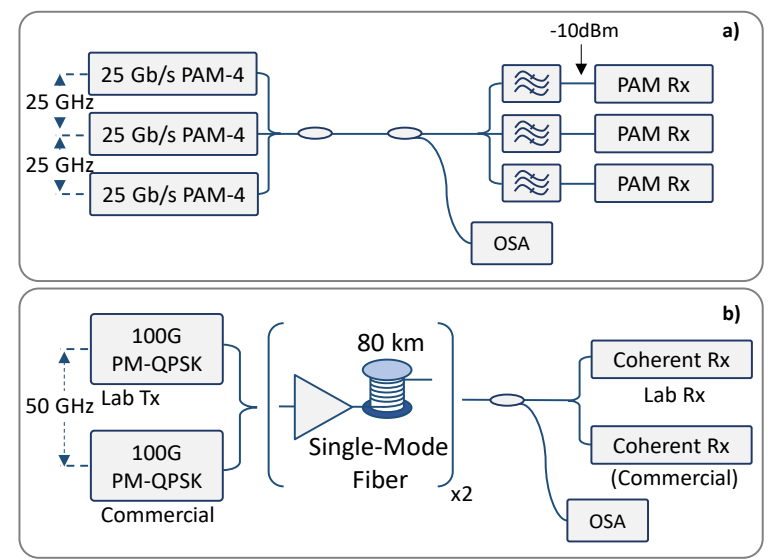

Fig. 6. Setup for PAM-4 (a) and QPSK (b) signals.
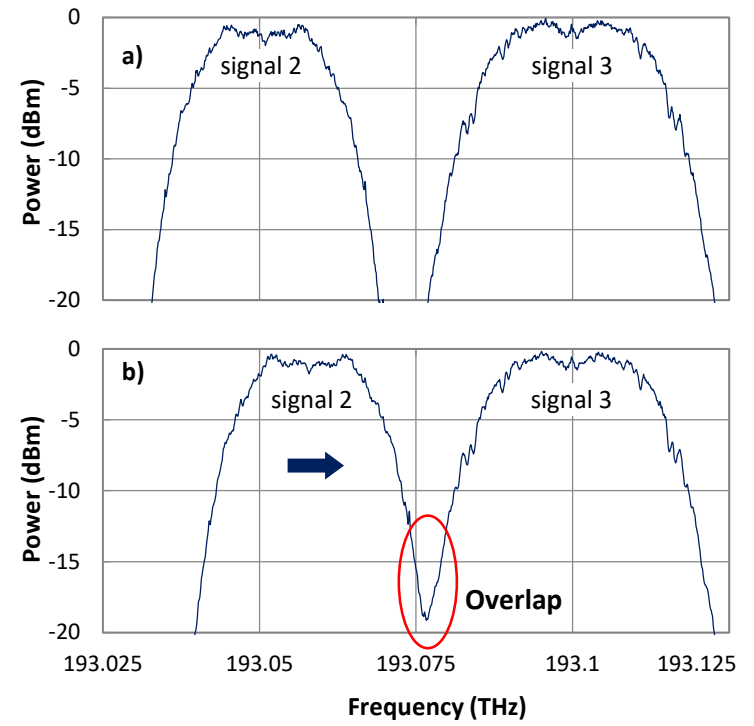

Fig. 7. Two QPSK optical signals under normal conditions (a) and when fLD causes overlap (b).

The first experiments that we carried out on such setups focused on quantifying the performance penalty introduced by misalignments and overlaps, specifically the impact of fLD on the BER. To this end, signals were generated and one of them simulated to drift to the right step by step to evaluate the degradation. In the case of PAM-4, we assumed steps of $200 \mathrm{MHz}$ up to $5 \mathrm{GHz}$ to introduce detuning with respect to the receiver optical filter, whereas for the QPSK case we used steps of $1 \mathrm{GHz}$ up to $25 \mathrm{GHz}$, emulating channel spacing reduction and producing signal overlapping (Fig. 7b). The obtained results are reported in Fig. 8a for PAM-4 systems and in Fig. 8b for QPSK systems. In view of the results, it is clear that misalignments and overlaps introduce significant performance penalty for both PAM-4 and QPSK signals. Specifically, noticeable BER increment is observed when fLD is above $3.6 \mathrm{GHz}$ in the case of PAM-4 and when channel spacing is reduced more than 10 $\mathrm{GHz}$ in the case of QPSK signals. 


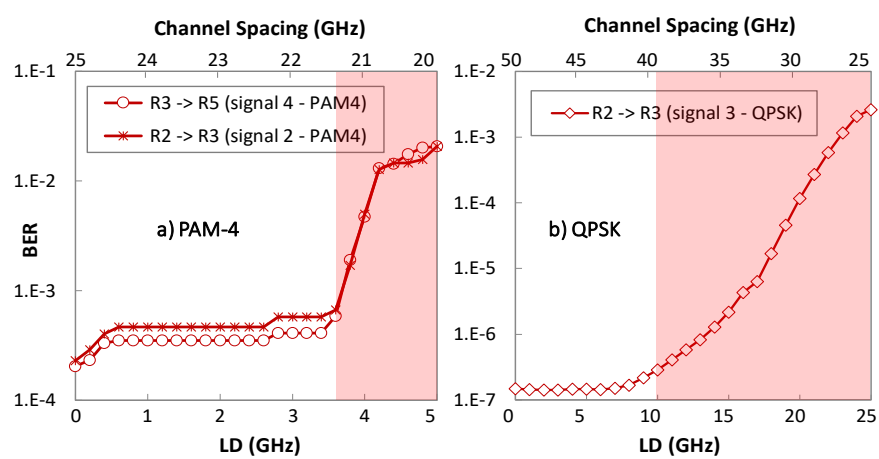

Fig. 8. BER degradation vs fLD and/or channel spacing for PAM-4 (a) and QPSK (b) systems.

Note that a different conclusion can be drawn in view of the results in Fig. 8; channel spacing can be reduced in about $3 \mathrm{GHz}$ for PAM-4 and $7 \mathrm{GHz}$ for QPSK signals without significantly affecting BER. However, such channel spacing reduction entails that fLD must be detected as soon as possible to avoid BER degradation of the channels. The next section evaluates the proposed optical signal tracking.

\section{B. Tracking individual optical signals}

Let us now focus on analyzing the optical signal tracking approaches in terms of tracking accuracy when they are applied to one single signal in an optical link, i.e., there are no neighbors nearby in the spectrum, like signal 4 in Fig. 1.

Firstly, we focus on the residual-based approach. We generated the expected signal following the procedure described in Section 3.B, assuming the characteristics of PAM-4 and QPSK signals. To make sure that the obtained spectra correspond to the measured PAM-4 and QPSK signals, we carried out several tests and calculate the residual signals in the case of signals without any fLD to investigate whether the residual signal oscillate with low magnitude around zero. Our criteria to choose a suitable expected signal was to make sure that in the proper operation regime, they look very much like the acquired spectra by OSAs.

Let us now focus on the selection of features for fLD estimation. For illustrative purposes, Fig. 9a plots the residual signals computed for PAM-4 signals when fLD varies from 0 to $+4.8 \mathrm{GHz}$ with $600 \mathrm{MHz}$ step size. As introduced in Section 3.B, we focus on the data points within the portion of the spectrum highlighted in blue color. Fig. $9 \mathrm{~b}$ plots the evolution of these points ( 3 data points when 1.2-GHz resolution OSAs are used) with respect to the magnitude of fLD. It is clear that as the simulated amount of fLD in the case of PAM-4 signals is small, a small width of the spectrum region is sufficient to be considered for the analysis.

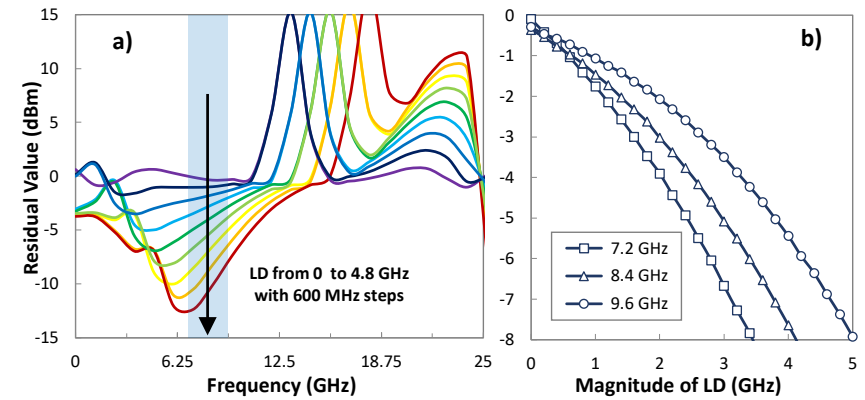

Fig. 9. Residuals for different fLD magnitudes for PAM-4 signals for optical spectra captured by a 1.2-GHz resolution OSA.

Fig. 10 shows the evolution of the considered data points for QPSK signals (18 data points when 1.2-GHz resolution OSAs are used), where larger amounts of fLD are measured; the residual traces are illustrated for magnitudes ranging from 0 to $24 \mathrm{GHz}$ with step size of $2 \mathrm{GHz}$. As shown, the residual signals at lower frequencies seem to be saturated and do not contain useful information for the fLD estimation of high magnitude. Therefore, in such scenarios, regions as wide as $20.4 \mathrm{GHz}$ are considered, where such region was partitioned into two bands to increase the estimation accuracy, resulting into two sets of features for estimation of small/medium (below $10 \mathrm{GHz}$ ) and large (up to $25 \mathrm{GHz}$ ) fLD magnitudes. The evolution of the features in both sets are plotted in Fig. 11. 


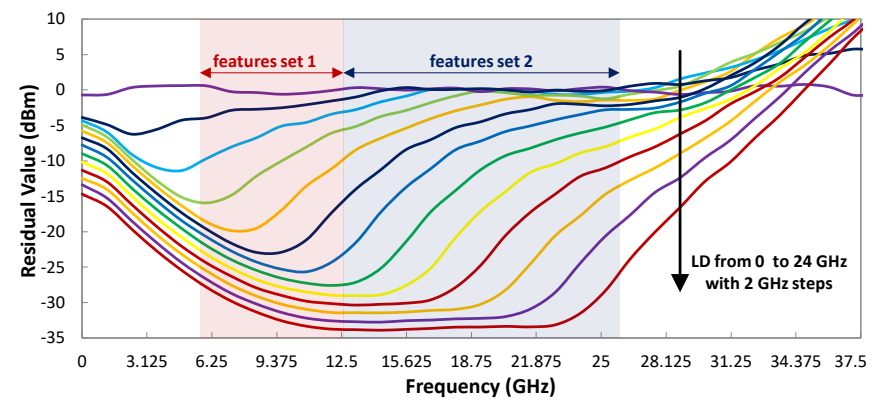

Fig. 10. Residuals for different fLD magnitudes for QPSK signals for optical spectra captured by a 1.2-GHz resolution OSA.

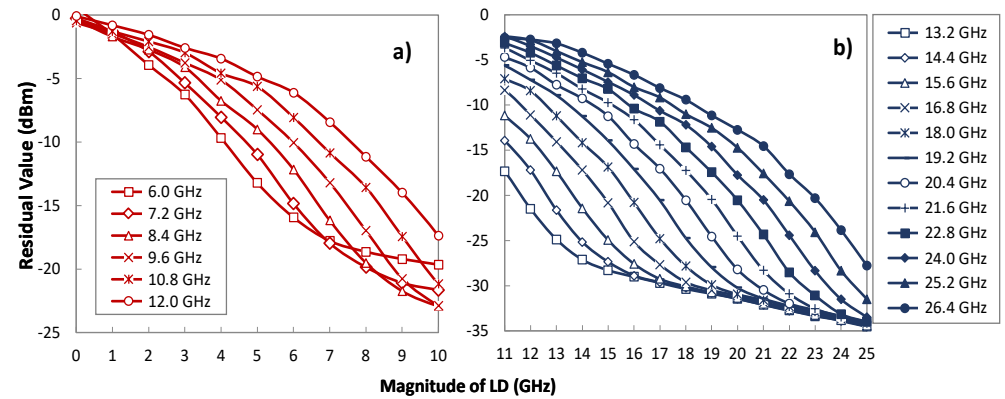

Fig. 11. Evolution of residuals with respect to the magnitude of fLD for QPSK signals using set 1 (a) and set 2 (b).

Once the selection of the features for the residual-based approach has been reviewed, let us investigate whether the two approaches for fLD estimation work well with acquisitions of OSAs of different resolutions. To this end, we emulated PAM-4 signal spectra acquired with 100-MHz resolution, whereas, for the experimental QPSK setup, a sequence of $\mathrm{C}$ - band spectra was acquired using a commercial OSA with the same resolution. Then, to analyze the impact of the resolution on the accuracy of the proposed approaches, a number of captures with coarser resolution, from $300 \mathrm{MHz}$ to $1.8 \mathrm{GHz}$, were generated by averaging powers from the original capture. The results are plotted in Fig. 12 for PAM-4 and QPSK signals; plots present the results for the Direct and the Estimated Features methods proposed for the feature-based approach, as well as for the Residual-based approach.
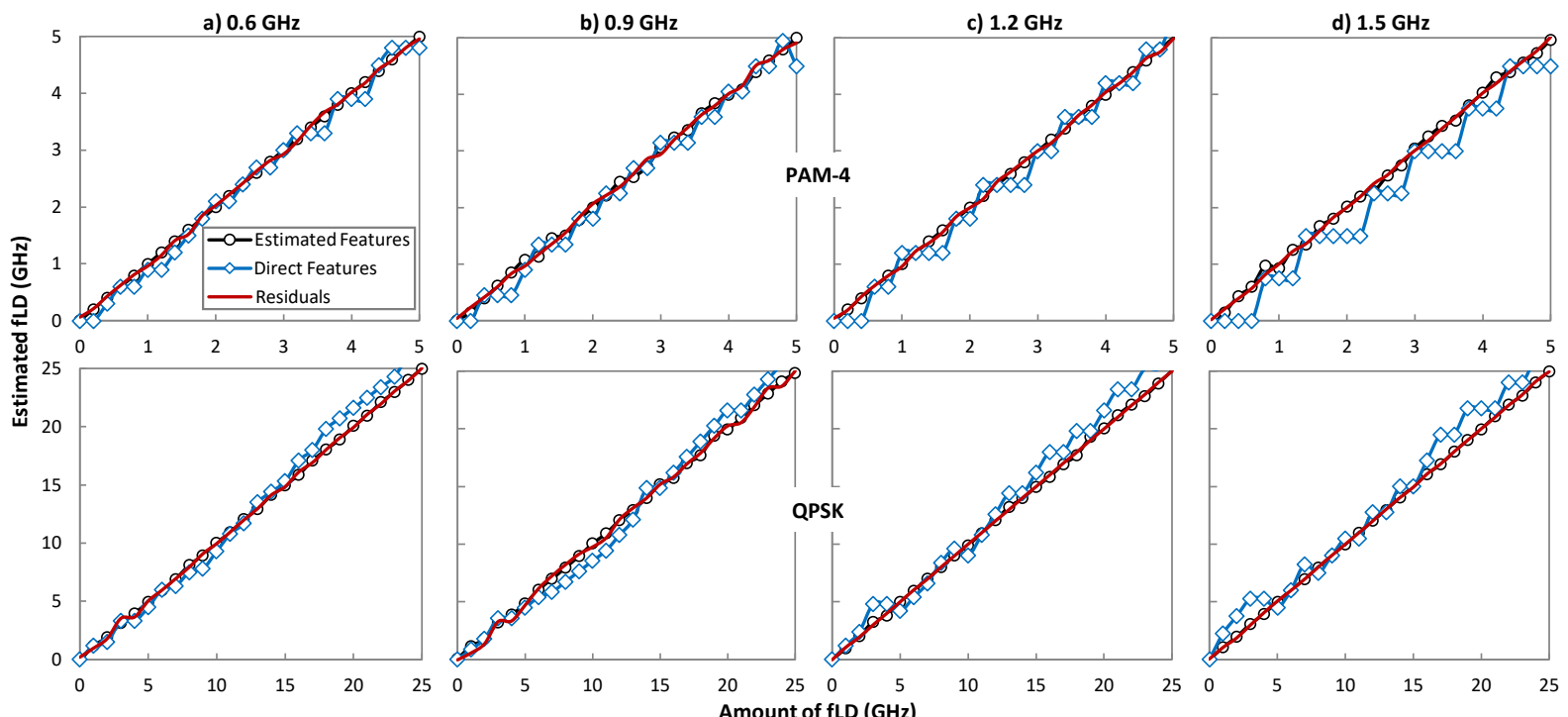

Fig. 12. Tracking accuracy for PAM-4 and QPSK signals for different OSA resolution.

As shown, the estimation accuracy of the Estimated Features method and the residual for both PAM-4 and QPSK signals is perfect regardless of the OSA resolution, while the accuracy of the Direct Features method degrades (notice the step-like behavior) as OSA of coarser resolution is considered. In order to numerically quantify the estimation accuracy of different approaches, Fig. 13 reports the mean relative estimation error of all three approaches for both PAM-4 and QPSK signals as a function of OSA's resolution. 


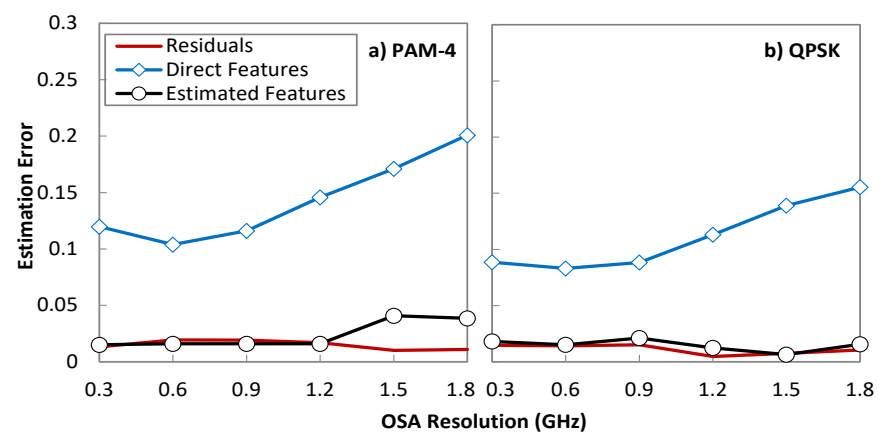

Fig. 13. Error against OSA's resolution for PAM-4 (a) and QPSK (b).

\section{Tracking multiple signals}

In this subsection, we consider a group of three neighboring signals, where the signal in the middle was affected by an fLD failure, similarly as signals 1, 2, and 3 in Fig. 1.

In the case of PAM-4, signals were spaced $25 \mathrm{GHz}$ and signal 2 was detuned towards signal 3 with steps of $200 \mathrm{MHz}$ down to the point where their spacing becomes $20 \mathrm{GHz}$. In the case of QPSK signals, the signals were spaced $50 \mathrm{GHz}$ and signal 2 was forced to move with steps of $1 \mathrm{GHz}$ down to the point where their spacing becomes $25 \mathrm{GHz}$. We are interested in evaluating whether the proposed approach enables reducing the channel spacing to reduce channel spacing, while monitoring the signals to detect overlapping.

The results for fLD estimation for signal 2 assuming PAM-4 signals showed the same accuracy as in the previous experiment for signal 4 . However, when the procedure is applied to analyze signal 3 , we observed that the accuracy was poor; the results are presented in Fig. 14a, where the estimation error is plotted as a function of the spacing between the two signals. These results are because of signal 2 partially overlaps signal 3 , which for coarse-granular OSA produces a significant loss of accuracy.

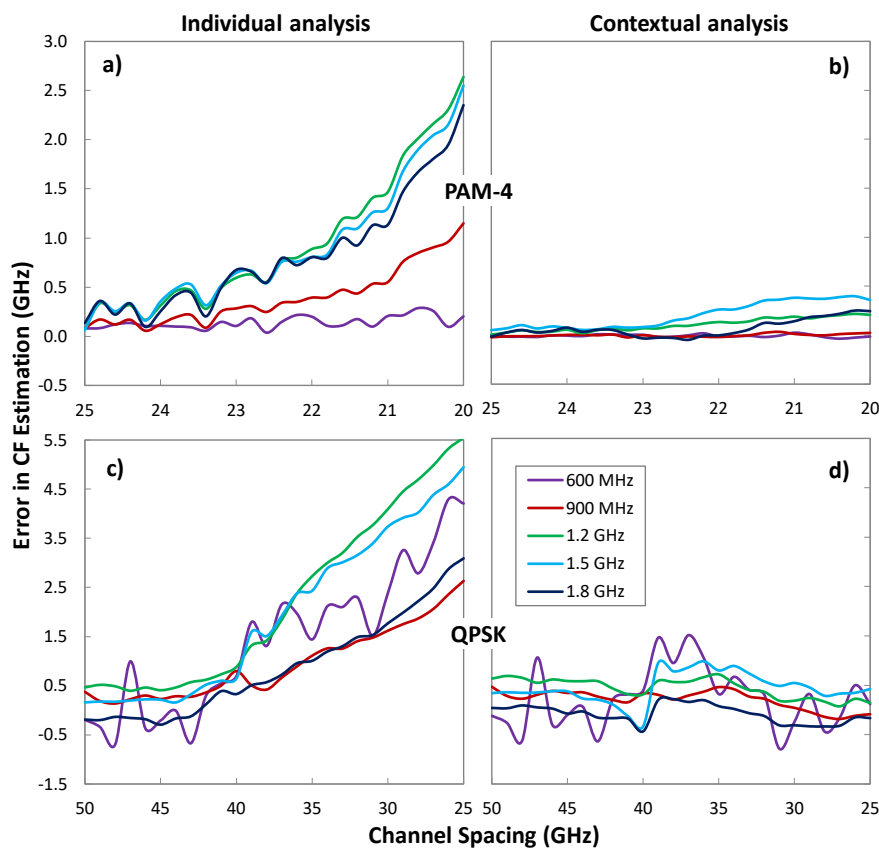

Fig. 14. Tracking accuracy vs. channel spacing for different OSA resolution with individual and contextual analysis for PAM-4 (a-b) and QPSK signals (c-d).

It is clear, in the view of these results that some contextual information needs to be added to the individual signal analysis, to take into account the detected fLD in a neighboring signal. Fig. 14b presents the results obtained when such contextual analysis is applied. The contextual expected signal generates residuals that counteract the effects of signal overlapping after finding that signal 2 presents some amount of fLD; this allows to preserve the accuracy observed in the first set of experiments. In fact, is that remarkable accuracy what enables reducing channel spacing, as any slight fLD can be detected and actions for laser retuning triggered. 
The same procedure was carried out for QPSK signals and the results are presented in Fig. 14c-d. As shown in Fig. 14c, when we apply the individual analysis approach, the estimation error increases and it falsely reports that signal 3 is moving, as soon as the overlap caused by the fLD in signal 2 becomes evident. Such estimation error vanishes when the contextual analysis approach is applied, as illustrated in Fig. 14d.

\section{CONCLUDING REMARKS}

The shorter distances of the metro segment enable for simpler and more cost-effective solutions, as compared to the core network. Here, FONs and direct-detection complementing coherent-detection transmission systems have been proposed as feasible solutions to extend the optical network toward the edges. To create FONs that are efficient in terms of spectrum resource utilization, optical channels should be placed very close to each other, which, in case of fLD, increases the risk of signal overlapping, as well as BER degradation in the case of directdetection transmission systems. For these reasons, a data analytics-based surveillance system has been proposed in this paper to detect anomalies and failures; it exploits the optical spectra of the lightpaths in a FON. The proposed surveillance system comprises of signal identification, classification, and tracking modules.

The main purpose of signal identification and classification modules is to check whether all the allocated lightpaths are placed in their expected spectral ranges; otherwise, the controller is notified. As for the optical signal tracking module, it is in charge of detecting and monitoring any fLD on those lightpaths found within their frequency ranges.

Two different approaches have been proposed: $i$ ) the features-based tracking approach that includes two different methods: direct features uses directly the features extracted from the optical signal, while the estimated features elaborates on the features and solves some of the issues of the direct approach; and ii) the residualbased approach, which uses a set of residual signals. The results showed that the residual-based approach provides accurate estimation of the CF of the signals using OSAs with resolution as coarse as $1.2 \mathrm{GHz}$. Such results proved the usefulness of the optical signal tracking module: $i$ ) to avoid misalignments of a transmitter with the optical filter of its corresponding receiver in direct-detection systems, and ii) to predict whether two neighboring lightpaths may overlap, disregarding their transmission technology. The latter greatly contributes to reduce channel spacing in about $3 \mathrm{GHz}$ for PAM-4 and $7 \mathrm{GHz}$ for QPSK signals without significantly affecting BER. Note that such channel spacing reduction entails notably increasing the capacity of the FON (21 additional optical channels in the case of PAM-4 and 13 in the case of QPSK signals).

Table 1. Comparison of different optical signal tracking methods

\begin{tabular}{|l|c|c|c|c|}
\cline { 4 - 5 } \multicolumn{1}{c|}{} & \multirow{2}{*}{$\begin{array}{c}\text { Pre- } \\
\text { processing }\end{array}$} & \multirow{2}{*}{$\begin{array}{c}\text { Training } \\
\text { required }\end{array}$} & \multicolumn{2}{|c|}{ Accuracy } \\
\cline { 4 - 5 } & Low & No & Low & - \\
\hline Direct Features & Medium & Yes & High & - \\
\hline Estimated Features & High & Yes & High & Very High \\
\hline Residual & & Hontextual \\
\hline
\end{tabular}

Table 1 summarizes the key differences of the proposed approaches. It can be concluded that the residualbased approach with its outstanding performance can be used to perform individual, as well as contextual optical signal tracking on direct-detection and coherent-detection systems. Note that those results complement those in [11] and [12] for SSON and clearly motivate the availability of OSAs in the data plane for accurate signal monitoring.

\section{ACKNOWLEDGEMENTS}

The research leading to these results has received funding from the European Commission for the H2020-ICT2016-2 METRO-HAUL project (G.A. 761727), from the AEI/FEDER TWINS project (TEC2017-90097-R), and from the Catalan Institution for Research and Advanced Studies (ICREA).

\section{REFERENCES}

[1] L. Velasco et al., "Saving CAPEX by Extending Flexgrid-based Core Optical Networks towards the Edges," (Invited Paper) IEEE/OSA Journal of Optical Communications and Networking (JOCN), vol. 5, pp. A171-A183, 2013.

[2] J. Zhang, J. Shi, and J. Yu, "The best modulation format for 100G short-reach and metro networks: DMT, PAM-4, CAP, or duobinary?" in proc. SPIE 2018. doi: 10.1117/12.2290622. 
[3] E. Archambault et al., "Design and simulation of filterless optical networks: Problem definition and performance evaluation," IEEE/OSA Journal of Optical Communications and Networking (JOCN), vol. 2, pp. 496-501, 2010.

[4] C. Tremblay et al., "Agile Optical Networking: Beyond Filtered Solutions," in Proc OFC, paper M1A.5, 2018.

[5] P. Lothberg, "Optical networking in DTAG's TeraStream project," in proc. OFC, paper W1K.1, 2016.

[6] Y. Zhao et al., "Channel Spacing Monitor Based on Periodic Training Sequence in DWDM System", IEEE/OSA Journal of Lightwave Technology (JLT), vol. 35, pp. 1422-1428, 2017.

[7] A. P. Vela et al., "BER Degradation Detection and Failure Identification in Elastic Optical Networks," IEEE/OSA Journal of Lightwave Technology (JLT), vol. 35, pp. 4595-4604, 2017.

[8] Z. Dong et al., "Optical Performance Monitoring in DSP-based Coherent Optical Systems," in Proc. IEEE/OSA Optical Fiber Communication Conference (OFC), 2015.

[9] Finisar. Flexgrid High Resolution Optical Channel Monitor (OCM) [On-line] www.finisar.com, 2017.

[10] M. Dallaglio et al., "Demonstration of a SDN-based spectrum monitoring of elastic optical networks," in Proc. IEEE/OSA Optical Fiber Communication Conference (OFC), 2017.

[11] A. P. Vela et al., "Soft Failure Localization during Commissioning Testing and Lightpath Operation [Invited]," IEEE/OSA Journal of Optical Communications and Networking (JOCN), vol. 10, pp. A27-A36, 2018.

[12] B. Shariati, M. Ruiz, J. Comellas, and L. Velasco, "Learning from the Optical Spectrum: Failure Detection and Identification [Invited]," IEEE/OSA Journal of Lightwave Technology (JLT), vol. 37, pp. 433-440, 2019.

[13] D. Rafique and L. Velasco, "Machine Learning for Optical Network Automation: Overview, Architecture and Applications," (Invited Tutorial) IEEE/OSA Journal of Optical Communications and Networking (JOCN), vol. 10, pp. D126-D143, 2018.

[14] A. P. Vela, M. Ruiz, L. Velasco, "Distributing Data Analytics for Efficient Multiple Traffic Anomalies Detection," Elsevier Computer Communications, vol. 107, pp. 1-12, 2017.

[15] L. Velasco et al., "Monitoring and Data Analytics for Optical Networking: Benefits, Architectures, and Use Cases," accepted in IEEE Network Magazine, vol. 33, pp. 100-108, 2019.

[16] L. Gifre et al., "Autonomic Disaggregated Multilayer Networking,” IEEE/OSA Journal of Optical Communications and Networking (JOCN), vol. 10, pp. 482-492, 2018.

[17] L. Velasco et al., "An Architecture to Support Autonomic Slice Networking [Invited]," IEEE/OSA Journal of Lightwave Technology (JLT), vol. 36, pp. 135-141, 2018.

[18] L. Velasco et al., "A Learning Life-Cycle to Speed-up Autonomic Optical Transmission and Networking Adoption," IEEE/OSA Journal of Optical Communications and Networking, vol. 11, pp. 226-237, 2019.

[19] M. Ruiz et al., "Knowledge Management in Optical Networks: Architecture, Methods and Use Cases [Invited],", IEEE/OSA Journal of Optical Communications and Networking, vol. 12, pp. A70-A81, 2020.

[20] B. Shariati et al., "Real-time Spectrum Surveillance in Filterless Optical Networks," in Proc. IEEE/OSA Optical Fiber Communication Conference (OFC), 2018.

[21] B. Shariati et al., "Optical Signal Tracking for Robust PAM-4 Deployment in Filterless Metro Network Scenarios," in IEEE/OSA Optical Fiber Communication Conference (OFC), 2019.

[22] METRO-HAUL project, "Deliverable D3.1: Selection of metro node architectures and optical technology options," [on-line: https://metro-haul.eu], 2018.

[23] N. Eiselt et al., "Performance Comparison of 112-Gb/s DMT, Nyquist PAM4, and Partial-Response PAM4 for Future 5G Ethernet-Based Fronthaul Architecture," IEEE/OSA Journal of Lightwave Technology, vol. 36, pp. 1807-1814, $\underline{2018 .}$

[24] J. Jobson, “Applied Multivariate Data Analysis: Regression and Experimental Design," Springer Texts in Statistics, 1991.

[25] C. Pulikkaseril et al., "Spectral modeling of channel band shapes in wavelength selective switches," OSA Optics Express, vol. 19, pp. 8458-8470, 2011.

[26] F. Cugini et al., "Push-pull defragmentation without traffic disruption in flexible grid optical networks," IEEE/OSA Journal of Lightwave Technology, vol. 31, pp. 125-133, 2013. 